\title{
Lymphorrhea in gastric cancer patients
}

Bandar Ali*

Division of Gastrointestinal Surgery, Department of Surgery, Prince Sultan Military Medical City, Riyadh, Saudi Arabia

\begin{abstract}
Introduction: Lymphorrhea defined as the leakage of serous lymphatic fluid in response to lymphatic duct disturbances. It could be resulted either due to malignant neoplasms, abdominal traumas, bacterial peritonitis, pelvic irradiation, peritoneal dialysis, or abdominal tuberculosis. It could also be from iatrogenic causes such as aortic surgery, spinal surgery, and lymph node dissection for various malignancies. Chylorrhea is a form of lymphorrhea, involving digested lipid products absorbed in the small intestine. The incidence of lymphorrhea after gastrectomy is unknown, while chylorrhea with D2 or less dissection is reportedly only $0-0.4 \%$. In this article we would like to review the incidence, management after gastrectomy for malignant pathology and stress in the different type of lymphatic leak.

Discussion: Anatomical variation in D1 or more dissection, does not typically cause injury to the main lymphatic trunks, but expected to lymphatic channels as oncological dissection is necessary for proper and the maximum number of lymph node retrieval for accurate staging. Lymphohrrea varies in presentation and severity according to the extent and location of the injury. One of these variations is chylous ascites as major lymphatic collecting system (trunk) is injured i.e., (cisterna chyli, or thoracic duct), which is more severe form of lymphorrhea and might need more aggressive form of treatment.

Conclusion: Care should be taken for Lymphorrhea after gastrectomy with D1 or more dissection, although it is very rare and more benign than chylorrhea. Some patients are more likely to have this complication than another due to their own risk factors. Multimodal treatment options are available, keeping these patients in fasting status followed by a low-fat diet without a necessity of TPN, with or without adding octreotide, which would be an effective therapy. In very rare event, severe and refractory cases, surgical intervention may be needed to control the leak.
\end{abstract}

\section{Introduction}

Lymphorrhea is defined as a serous or yellow-colored abdominal fluid iso-osmotic to interstitial one, not containing chyle; thus fluid and serum triglycerides levels are nearly equal. An injury up to the level of pre-nodal collecting lymphatic causes this complication. Finally, radionuclide lymphangiography may be therapeutic in cases of lowoutput chyle leakage, since nano-colloids with a diameter of $80 \mathrm{~nm}$ may act as an embolus inside the lymphatic vessels $[1,2]$. This should be differentiated from chylorrhea and its synonyms should be defined as a milky, sterile, odorless and rich in triglycerides peritoneal fluid [2-4]. We propose that injury at any point distal to the post nodal collecting lymphatics and not only to the classically described cisterna chyli, can cause chylorrhea.

Postoperative lymphorrhea has been observed after retroperitoneal lymphadenectomy for urinary oncological surgery [5], portal system decompression [6], abdominal aortic surgery [7], renal transplantation [8], and after arthrodesis using an anterior approach to the spine [9]. The incidence of lymphorrhea was approximately $7.4 \%$ after oncological abdominal surgery [10], however, only a few cases of lymphorrhea after radical gastrectomy for gastric cancer have been reported $[11,12]$. Lymph node metastases occur during the early stages of gastric cancer, and regional lymphadenectomy is recommended as a part of radical gastrectomy [13]. The vast majority of these cases were subclinical and recovered spontaneously, but in some instances they can persist for long period of time causing significant morbidity (Figure 1-3).

\section{Discussion}

In some patients who are considered at high risk for the lymphatic leak as they had the underlying liver disease either alcoholic cause or viral hepatitis cause i.e. portal hypertension and stigmata of chronic liver diseases. Even with meticulous dissection lymphatic leakage cannot be avoided as lymph node dissection for oncological resection needed. Preoperative optimization liver parameter as much as possible will reduce the incidence of post op lymphorrhea. Another theoretical believed cause of lymph leak is the extensive usage of electrocautery might lead to improper sealing and clipping or ligation may lead to better outcomes (Figure 4).

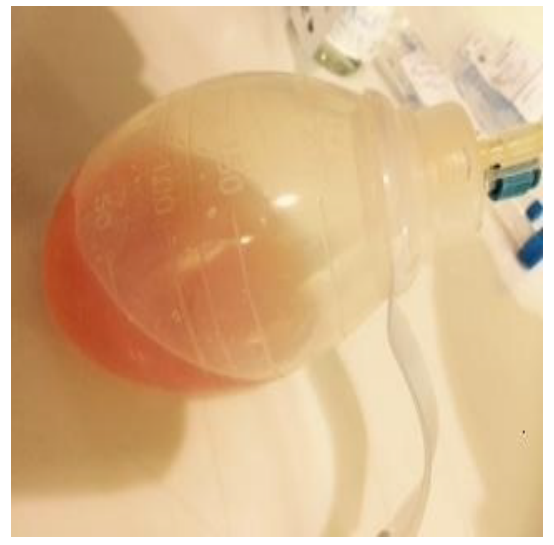

Figure 1. Serous output from drain (lymphorrhea)

Serous appearance of the drained fluid, highly suggestive for lymphorrhea formation

Correspondence to: Bandar Ali, M.D., Division of Gastrointestinal Surgery, Department of Surgery, Prince Sultan Military Medical City, Riyadh, Saudi Arabia, Tel: +966-555310007, E-mail: biaa1003@yahoo.com

Key words: lymphorrhea, gastric cancer, gastrectomy, laparoscopic gastrectomy

Received: April 02, 2016; Accepted: May 27, 2016; Published: May 30, 2016 


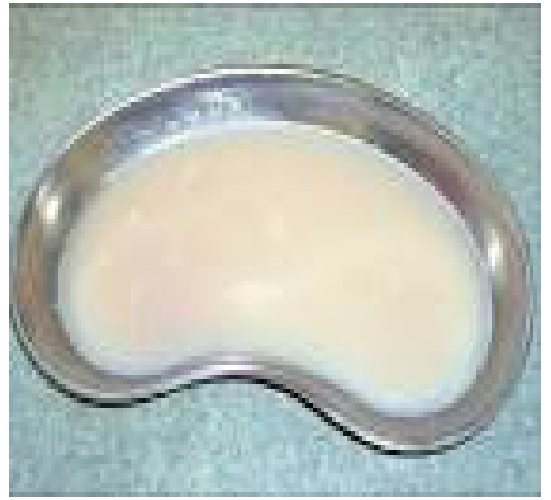

Figure 2. Typical chyle

Serous appearance of the drained fluid, highly suggestive for lymphorrhea formation

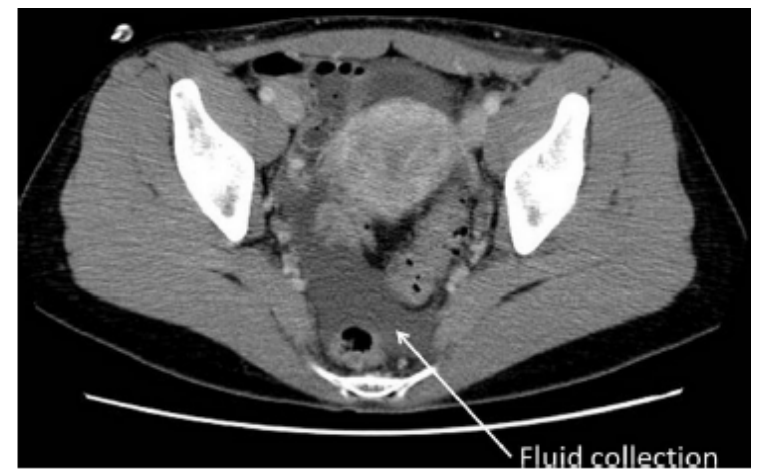

Figure 3. A contrast-enhanced CT scan shows a little ascites at pelvic space (white arrow)

Abdominal CT scan of the patient, disclosing large amount of abdominal fluid successfully drained percutaneously under $\mathrm{CT}$ guidance. The correct placement of the percutaneous catheter is shown



Figure 4. Upper body main lymphatic drainage system.

Causes of lymphorrhea after gastric cancer surgery are numerous, and the main cause is the extensive surgical procedure technique which is needed to maintain oncological principle.

Diagnostic methods are clinical, nuclear scan and radiological. Radiological imaging may be necessary to ensure no other missed injuries.

The lymphatic leaks from the stumps of the small and invisible lymph vessels into the free abdominal cavity resulting in lymphorrhea [13]. The more extensive the lymph node dissected, the higher incidence of lymphorrhea will be. It has been reported in literature that the incidence of lymphorrhea is approximately $1.2-3.0 \%$ after retroperitoneal mesenteric vessel pedicle lymph node dissection.

Some studies suggested that electro cautery usage result in higher incidence of lymphatic leak than in those ligation was used.

The electro cautery cannot completely seal the largely disrupted lymph vessels and lymph vessels with large drainage volumes. The scars on the lymph vessel stumps after cauterization can disintegrate due to the increased lymph stump pressure [13].

The prevention of lymphorrhea after gastric cancer surgery is most important than treatment especially during lymph node dissection for gastric cancer, the cistern chyli or the surrounding lymph vessel network can be injured, network around the abdominal aorta, inferior caval vein, and mesenteric vessels can be easily injured [5].

The treatment of lymphorrhea most of the time after gastric cancer surgery is conservative.TPN can dramatically decrease the lymph flow in the thoracic duct from $220 \mathrm{ml} /(\mathrm{kg} \mathrm{h} / 1)$ to $1 \mathrm{ml} /(\mathrm{kg} \mathrm{h} / 1)$ [14].

Moreover, TPN can restore the nutritional deficits and balance the metabolic impairments imposed by long-standing lymphorrhea. Therefore, TPN is essential in the no operative management of lymphorrhea [13].

Some recent studies suggested promising result from somatostatin usage. The mechanism by means of octreotide affects lymphorrhea is unknown. It has also been speculated that somatostatin.

Improves chylous ascites by inhibiting lymph fluid excretion through specific receptors found in the normal lymphatic vessels of the intestinal wall $[15,16]$. However, somatostatin receptors have been found in lymphatic tissues also outside the gastrointestinal tract [16], so it is possible that there should be a direct action on lymphatic vessels. Somatostatin has previously been shown to decrease the intestinal absorption of fats, lower the triglyceride concentration in the thoracic duct, and attenuate lymph flow in the major lymphatic channels.

Formation of the interstitial fluid as blood passes through the capillaries some of the water-soluble material materials and white cells escape through the capillary walls. This liquid fills the space between the tissue cells and constitutes the interstitial fluid, or tissue fluid. Part of the interstitial fluid is absorbed back into the capillaries. The rest passes into the lymph vessels to become lymph.

The lymphatic system has three main functions. First, it returns excess interstitial fluid (also called tissue fluid) to the blood. Of the fluid that leaves the capillaries, about 90 percent is returned. The 10 percent that doesn't return becomes part of the tissue fluid that surrounds the tissue cells. Small protein molecules may "leak" through the capillary wall and increase the osmotic pressure of the interstitial fluid (see osmosis). This further inhibits the return of fluid into the capillaries, and fluid tends to accumulate in the tissue spaces. If this continues, blood volume and blood pressure decrease significantly and the volume of tissue fluid increases, which results in edema (swelling). Lymph capillaries pick up the excess interstitial fluid and proteins and return them to the venous blood. After the fluid enters the lymph capillaries, it is called lymph.

The second function of the lymphatic system is the absorption of fats and fat-soluble vitamins from the digestive system and the subsequent transport of these substances to the venous circulation. The mucosa that lines the small intestine is covered with fingerlike 
projections called villi. There are blood capillaries and special lymph capillaries, called lacteals (see below), in the center of each villi. The blood capillaries absorb most nutrients, but the fats and fat-soluble vitamins are absorbed by the lacteals. The lymph in the lacteals has a milky appearance due to its high fat content and is called chyle.

The third and probably most well-known function of the lymphatic system is defense against invading microorganisms and disease. In other words, the lymph system is part of the immune system. Lymph nodes and other lymphatic organs filter the lymph to remove microorganisms and other foreign particles. Lymphatic organs contain lymphocytes that destroy invading organisms

Then, the fluid is propelled further following the route: initial lymphatics $\rightarrow$ pre-nodal collecting lymphatics $\rightarrow$ lymph nodes $\rightarrow$ post-nodal collecting lymphatics $\rightarrow$ lymphatic trunks $\rightarrow$ cisterna chyli $\rightarrow$ thoracic duct. From the level of collecting lymphatics, lymph propulsion through the lymph nodes into the ducts, is favored by the presence of contractive smooth muscle and valves. Significant changes in lymph concentration occur as the fluid passes through the various components of the lymphatic system. It becomes concentrated along the contracting lymphangion (the segment of a collecting lymphatic between valves) segments, possibly due to water filtration across the vessel wall, while protein concentration decreases during its residence in the lymph nodes from osmotically driven fluid exchange with nodal blood vessels and phagocytosis by white cells. Thus, the sampling point of lymph fluid (eg. prenodal versus postnodal lymphatics, lymph node versus duct, etc) strongly affect molecule uptake data reported in the literature.

Based on the above, any disruption of the lymphatic system at the level of initial lymphatic collecting system causes accumulation of isoosmotic fluid. Triglycerides fluid levels are nearly equal to serum level. As more distal to the collecting lymphatics the disruption occurs, the more concentrated the absorbed molecules are; hence triglycerides fluid levels are higher than serum level. At which level of the lymphatic system, the triglycerides fluid levels become doubled to the interstitial one, it is not clear.

\section{Conclusion}

Lymphorrhea is different from Chylorrhea which is more serious (transudate) and more benign form than chylorrhea. Although it is rare after gastrectomy procedure but incidence expected to rise as more lymph node dissection is done and patient's underlying diseases mainly portal hypertension. Prevention is the most important treatment modality.

\section{References}

1. Ablan CJ, Littooy FN, Freeark RJ (1990) Postoperative chylous ascites: diagnosis and treatment. A series report and literature review. Arch Surg 125: 270-273. [Crossref]

2. Wu CW, Hsieh MC, Lo SS, Lui WY, P'eng FK (1996) Results of curative gastrectomy for carcinoma of the distal third of the stomach. J Am Coll Surg 183: 201-207.[Crossref]

3. Endo M, Maruyama K, Kinoshita T, Sasako M (1994)Chylous ascites after extended lymph node dissection for gastric cancer. Jpn J Gastroenterol Surg 27: 917-921.

4. Song CY, Park BS, Kwon SJ, Park YS, Kwon OJ, et al. (1997) Reasonable time for removal of the nasogastric tube after a radical gastrectomy. J Korean SurgSoc 53: 809816.

5. Ferrigni RG, Novicki DE (1985)Chylous ascites complicating genitourinary oncological surgery. J Urol 134:774-776.

6. Maywood BT, Goldstein L, Busuttil RW (1978) Chylous ascites after a Warren shunt. Am J Surg 135: 700-702.[Crossref]

7. Williamson C, Provan JL (1987) Chylous ascites following aortic surgery. Br J Surg 74: 71-72.[Crossref]

8. Liu CJ, Lo SC, Kao JH, Tseng PT, Lai MY, et al. (2006) Transmission of occult hepatitis B virus by transfusion to adult and pediatric recipients in Taiwan. J Hepatol 44: 39-46.[Crossref]

9. Shen YS, Cheung CY, Nilsen PT (1989) Chylous leakage after arthrodesis using the anterior approach to the spine. Report of two cases. J Bone Joint Surg Am 71: 12501251.[Crossref]

10. Kaas R, Rustman LD, Zoetmulder FA (2001) Chylous ascites after oncological abdominal surgery: incidence and treatment. Eur J Surg Oncol 27: 187-189. [Crossref]

11. Iankin AV (2006) [Tachocomb plates efficacy in prevention of lymphorrhea in radical operations for gastric cancer]. Khirurgiia (Mosk): 44-46.[Crossref]

12. Tanaka K, Ohmori Y, Mohri Y, Tonouchi H, Suematsu M, et al. (2004) Successful treatment of refractory hepatic lymphorrhea after gastrectomy for early gastric cancer, using surgical ligation and subsequent OK-432 (Picibanil) sclerotherapy. Gastric Cancer 7: 117-121. [Crossref]

13. Sheng-Zhang L, Hong-Fei T, Zhong-Lin N, Yao-Jun Y, Tao Y, et al. (2009) Treatment and prevention of lymphorrhea after radical gastrectomy of gastric cancer. $J$ Cancer Res Clin Oncol 135: 613-616.[Crossref]

14. Aalami OO, Allen DB, Organ CH Jr (2000) Chylous ascites: a collective review. Surgery 128: 761-778.[Crossref]

15. Shapiro AM, Bain VG, Sigalet DL, Kneteman NM (1996) Rapid resolution of chylous ascites after liver transplantation using somatostatin analog and total parenteral nutrition. Transplantation 61: 1410-141. [Crossref]

16. Reubi JC, Horisberger U, Waser B, Gebbers JO, Laissue J (1992) Preferential location of somatostatin receptors in germinal centers of human gut lymphoid tissue. Gastroenterology 103: 1207-1214. [Crossref]

Copyright: (C2016 Ali B. This is an open-access article distributed under the terms of the Creative Commons Attribution License, which permits unrestricted use, distribution, and reproduction in any medium, provided the original author and source are credited. 